\title{
Las palabras que definen: Análisis del discurso ecológico
}

\begin{abstract}
Resumen
Este artículo examina los diversos lenguajes que se usan en el ámbito de la ecología, a partir de las palabras y conceptos bíblicos vinculados a esta temática. Lo hace de a pares: creación/naturaleza, bienes/recursos, cuidado/conservación, dominar/laborar. Las palabras "vida" y "destrucción" son transversales en todas las otras definiciones. También considera sus implicaciones en el ámbito de la economía capitalista imperante y las idolatrías que conlleva.
\end{abstract}

\begin{abstract}
This article examines the various languages used in the field of ecology, from the words and biblical concepts linked to this theme. It does it in pairs: creation/nature, goods/resources, care/maintenance, domination/ tillage. The words "life" and "destruction" are cross-cutting in all the other definitions. It also considers its implications at the level of the prevailing capitalist economy and the idolatry that entails.
\end{abstract}

\section{Introducción}

Siendo la Biblia un "libro de texto", un mensaje escrito, el uso y significado de las palabras es fundamental. Hay un "lenguaje bíblico" que le da a las palabras un nuevo sentido, al incorporarlas en un contexto de "revelación", sea cual sea el valor teológico que demos a este término, él mismo objeto de las diversas significaciones y posiciones teóricas en el entorno de lectura bíblica.

Por otro lado, también la ciencia y los movimientos sociales crean su propio lenguaje, porque sin ello no podrían nombrar y calificar sus hallazgos, objetos y logros, sus propias "revelaciones". Lo hace también la economía capitalista, para justificar sus posicionamientos. El lenguaje bíblico, el de la ciencia y el de la economía, así como el de los movimientos sociales no coinciden. Esto es claro ya desde hace varios siglos, siendo el ejemplo de la "evolución" el más notable y que más repercusión histórica ha tenido. Lo mismo podemos decir sobre las cuestiones de género, donde 
el lenguaje bíblico, teñido de patriarcalismo, ha de ser revisado a partir de la conciencia de su incidencia en los juicios y prejuicios, en las relaciones personales y en las prácticas sociales, en la creación de la cultura discriminatoria de la cristiandad.

Esto también es cierto para las ciencias del ambiente y para los movimientos sociales y políticos que expresan sus preocupaciones sobre lo ecológico. El lenguaje, las implicaciones lexicales, las proyecciones semánticas de las diversas corrientes que se dan en esta temática, con sus distintos enfoques y énfasis, han creado su propia lengua, incluso sus diversos "dialectos" en torno de la conciencia del complejo problema de la vida y condición del planeta a partir de la cada vez más notable influencia de la actividad humana. Pero no es solo una cuestión de palabras y traducciones, sino que también hay una diferenciada concepción del mundo, de la realidad (o varias concepciones, para mejor decir) que, al crear sus propios lenguajes, ponen de manifiesto posiciones de fe y supuestos ideológicos subyacentes. Detrás de cada palabra, de cada significante y significado, se esconden planteos, visiones y hasta políticas distintas. En ese sentido la fe bíblica entra en el diálogo ecológico con sus propios aportes, con sus convicciones e inevitables parcialidades. Un breve examen de ciertos términos nos servirá para notar algunos de estos elementos alternativos. Los presentamos de a pares, no para señalar contradicciones (aunque en algunos casos las hay) sino para mostrar de qué manera inciden en nuestra respuesta ante lo que podríamos llamar "el problema ecológico".

\section{Creación/naturaleza}

"En el principio creó Dios los cielos y la tierra" (Gén 1:1). Así comienza nuestra Biblia. Lo primero que sabemos de Dios es que es creador. $Y$ lo primero que sabemos de los cielos y la tierra es que han sido creados, y que esa creación es buena. Y mientras el Génesis destaca la bondad de la obra creada, los Salmos apuntan poéticamente a su belleza (por ejemplo, Sal 19:1-6).Esto definirá el relato bíblico desde ese momento hasta su final en el libro de Apocalipsis, donde nuevamente la potencia creadora de Dios es expuesta radicalmente en un cielo nuevo y una nueva tierra, que guarda significativas similitudes y diferencias con la descripción del primer paraíso. $\mathrm{Y}$ así tanto en la primera creación como en su renovación final hay salud, árbol de la vida, ríos que corren, luz emanada del propio Dios; pero por otro lado la nueva creación ya no es un jardín, un paraíso agrario, sino que es una ciudad. La labor humana, creadora de ciudades, es ahora asumida por la deidad. Una ciudad donde lo humano histórico no ha sido borrado (están los nombres de los patriarcas, de los apóstoles, los pueblos que han salido de la "gran tribulación"), pero todo es tocado con una nueva vida, donde "ya no habrá muerte, ni habrá más llanto, ni clamor, ni dolor; porque las primeras cosas pasaron" (Ap 21:4). 
En tanto creador y sustentador, en tanto dueño de todo lo creado - "De YHVH es la tierra y su plenitud; el mundo, y los que en él habitan" (Sal 24:1) - Dios entrega esa, su posesión, al ser humano para que la guarde, trabaje, haga multiplicar. Pero siempre, en última instancia, ha de pedir cuentas sobre lo que se hace con esa creación, incluyendo la actitud ante los otros seres humanos. No nos extenderemos en este asunto porque ya es objeto de otros artículos de este mismo número de RIBLA (y de otros números anteriores). Lo que quiero destacar acá es el hecho de que lo creado no es "anónimo". Hay quien lo ha creado (sea cual sea el significado y proceso que le demos a esa palabra) y ese creador sigue atento a lo que pasa con su obra. Para poner una metáfora artística, es como quien escribe una obra de teatro, que convoca a actores y actrices para que la interpreten y les da la libertad para que pongan en ello su propia creatividad, pero que no se desentiende de lo que éstos hacen, sino que continuamente vuelve sobre los énfasis que dejó en su escrito, revisa su texto, hace sugerencias, y finalmente evalúa cómo ha sido expuesto su mensaje.

Por otro lado, la Biblia no conoce la palabra "naturaleza" en el sentido que tiene en el discurso moderno. De hecho, el idioma hebreo no posee una palabra semejante ni tiene ese concepto. Para lo que nosotros llamamos naturaleza siempre se señala la creación, la dinámica de lo creado, que alude directa o indirectamente al creador y al sentido que le ha dado. Tanto es así que la palabra griega fysis (que es lo que normalmente traducimos por "naturaleza") no figura en la traducción al griego de los libros de la Biblia hebrea, sino sólo en los libros griegos de la Septuaginta, (3 y 4 Mac y Sab), y esto solo ocasionalmente.

En el Nuevo Testamento tampoco encontraremos un uso fluido de la palabra y concepto de fysis. La palabra y sus derivados solo aparecen 14 veces, y salvo dos excepciones, (Santiago y 1 Pedro) solo en los textos paulinos. Esto contrasta con otros escritos del judaísmo helénico de su tiempo, ya que el uso es abundante en Filón de Alejandría y en Flavio Josefo. ${ }^{1} \mathrm{Su}$ ausencia en los Evangelios la pone fuera del lenguaje de Jesús, al menos en estos registros. En contraste, la palabra ktisis (creación, lo creado) se da en 19 ocasiones, mayormente en Pablo, pero también en el Evangelio de Marcos, en Hebreos, en las dos cartas de Pedro y en Apocalipsis.

Dado que el mayor uso de ambas aparece en Pablo, me detendré a mirar allí. Para los escritos paulinos lo "físico" es de alguna manera "lo dado", lo establecido por el uso o por la condición de las cosas. Así, en Romanos 1:26 Pablo señalará el camino de la corrupción de las costumbres paganas en la Roma imperial (según el ideario judío en ese sentido) cuando dice que "sus mujeres cambiaron el uso natural por el que es contra naturaleza" (fysikos y para fysin, respectivamente). Curiosamente usará

\footnotetext{
${ }^{1}$ Ver Fysis en el Theological Dictionary of the New Testament, Vol. IX. G. Kittel y G. Friedrich (eds). Grand Rapids, Mi: Wm. B. Eerdmans Publishing Company, 1974.
} 
el mismo contraste al hablar de la inserción de los pueblos gentiles en la redención mesiánica, ya que mientras Israel se encuentra en esa condición "según su naturaleza" (kata fysin), los pueblos gentiles han sido injertados "para fysin" (contra la naturaleza).

La idea de naturaleza como el uso o costumbre mayormente aprobado aparece en Romanos 2:14, donde se dice que los gentiles hacen "por naturaleza" lo que manda la ley, o en 1 Corintios 11: 14 donde señala el uso del cabello en el varón o en la mujer. Otro uso paulino emplea fysis para indicar lo externo, la apariencia, lo "físico" en contraste con la identidad totalizadora de la fe (Rom 2:27). Y, siguiendo un uso que viene del griego pre-helenístico, fysis indica lo hereditario, lo que proviene de nacimiento (Rom 2:27; Gál 2:15; Ef 2:3). Finalmente, naturaleza indica, como en nuestro uso, la condición de los seres, su especie o clase (naturaleza humana, naturaleza divina) en Gálatas 4:8, que es el uso que le dan los textos de Santiago (3:7) y 2 Pedro (1:4).

Como puede verse, está ausente la idea de "naturaleza", como concepto amplio que indica lo existente, o, como lo define el Diccionario de la lengua española en sus primeras dos acepciones: "1. Principio generador del desarrollo armónico y la plenitud de cada ser, en cuanto tal ser, siguiendo su propia e independiente evolución. 2. Conjunto de todo lo que existe y que está determinado y armonizado en sus propias leyes". ${ }^{2}$ Así, el cuidado y preservación de "la naturaleza" sería, simplemente, mantener lo existente, preservar los usos y costumbres dados, reconocer la clase o especie de las cosas. A lo sumo indicaría la actualización de lo que ya está "en potencia" en cada ser. La Biblia, en cambio, y no solo por su época y cultura, sino porque hace a su concepción del mundo, no conoce el concepto de "leyes de la naturaleza, y menos aún el de "leyes económicas". El sostén, dinámica y subsistencia de lo existente se deben a la voluntad de Dios. Por eso lo que llamamos "milagro" en el relato bíblico no es un hecho "contra la naturaleza" sino la creación obedeciendo a su creador. ${ }^{3}$

Sin duda la idea de una naturaleza guiada por "leyes" contrasta con el concepto dinámico de creación, y sobre todo de la participación humana en la creación y en la renovación de la creación. ${ }^{4}$ Más allá de los textos que se refieren a la creación como obra de Dios, citando a Génesis, quizás el más significativo es nuevamente Pablo, especialmente en Romanos 8: 1923. Aquí se muestra toda una dinámica de la creación, su condición de ser vivo. Esta creación "ha sido atada a la vanidad de la corrupción", pero a la vez expresa su deseo de liberación. Es una creación que vive su cautive-

\footnotetext{
${ }^{2}$ Diccionario de la lengua española. Real Academia Española, Actualización 2017. En adelante DLE. Accesible en http: / / dle.rae.es

${ }^{3}$ Es esclarecedor el episodio de Lc 8:23-25, cuando Jesús calma la tempestad. La exclamación de los discípulos: "Y atemorizados, se maravillaban, y se decían unos a otros: ¿Quién es éste, que aun a los vientos y a las aguas manda, y le obedecen?" solo podía tener una respuesta: Dios mismo. ${ }^{4}$ La propia ciencia, a partir de la física quántica, está revisando este concepto, así como lo hacen algunas visiones ecologistas más de avanzada.
} 
rio "en esperanza" de redención, redención que se expresa en la "libertad gloriosa de los hijos e hijas de Dios", redención que es "redención de los cuerpos". Es una creación que sufre, pero sus dolores no son de agonía sino de parto, señal de una nueva creación que se está gestando dentro de ella. Esta creación ya es una nueva realidad "en Cristo" (2Co 5:17). Por eso "el conjunto de todo lo que existe" no es la naturaleza sino la creación, que ha de ser puesto bajo el señorío liberador del Mesías, que es partícipe y primogénito de esta creación (Col 1:15-23).

La creación no se libera si no se libera al ser humano. Las condiciones de cautividad y corrupción de lo creado son las condiciones de cautividad y corrupción de los propios seres humanos. Como ya señalamos, el mensaje bíblico no conoce una idea de naturaleza autosuficiente o estática, con sus propias leyes. Conoce, en cambio la dinámica liberadora del ser humano en el paradigma mesiánico, que es redención de todo lo creado, y anuncio de la renovación total, un cielo nuevo y una nueva tierra, donde lo histórico humano sobrevive, liberado de sus ataduras y muerte.

\section{Bienes/recursos}

Justamente por ser parte de la creación en la que todo es bueno, los elementos de la creación de los que dispone el ser humano son "bienes". Lo creado es bueno, y bueno en gran manera (Gén 1: 31). El sentido de la participación humana en esa creación es el de hacer uso y multiplicar esos "bienes". Así, la bendición de Dios señala: "te hará YHWH sobreabundar en bienes (tobah), en el fruto de tu vientre, en el fruto de tu bestia, y en el fruto de tu tierra, en el país que YHWH juró a tus padres que te había de dar" (Deut 28:11). Este uso de lo creado y disponible para el ser humano como "bienes" se distingue de cuando se refiere simplemente a propiedad o posesiones, para los cuales se usa en hebreo la raíz $r k s h$, o bz cuando son despojo de guerra, o 'shr, en referencia a las riquezas. En ese sentido tanto la LXX como el Nuevo Testamento suelen recurrir a la palabra huparjon.

Por el contrario, el desarrollo económico en general, pero especialmente la contabilidad del capitalismo, los "bienes" son vistos como "recursos". Lo creado no son sólo los "bienes contables", sino fundamentalmente "recursos": recursos materiales, recursos naturales, recursos financieros, y, cabe señalar especialmente, recursos "humanos". Es decir, la totalidad de lo creado, sea por Dios o por la propia actividad humana, es objetivada como instrumental, dado que un recurso es siempre un medio para lograr un fin. Nuevamente recurrimos al Diccionario de la lengua española: Recurso, acepción 2: "Medio de cualquier clase que, en caso de necesidad, sirve para conseguir lo que se pretende". Y más explícitamente en las acepciones 7, y 8: "7. Conjunto de elementos disponibles para resolver una necesidad o llevar a cabo una empresa. Recursos naturales, hi- 
dráulicos, forestales, económicos, humanos. 8. Expedientes, arbitrios para salir airoso de una empresa" ${ }^{5}$

Es decir, la idea de "la naturaleza como recurso" es opuesta al concepto de "lo creado como bien". La acepción 6 del diccionario: "Bienes, medios de subsistencia", emplea la palabra bienes en el sentido de posesiones, pero nuevamente los define como "medios". Algo similar ocurre en la definición que el DLE nos da de "bien" en el sentido económico: “4. m. Econ. Todo aquello que es apto para satisfacer, directa o indirectamente, una necesidad humana". Es decir, la creación no es el sujeto de la actividad humana, sino que es cosificada en función de lo que "quiere llevar a cabo una empresa", "para salir airoso de una empresa". Los bienes no son vistos como parte de una creación integral, sino en función de una satisfacción. ¡Y en esa cosificación son incluidos los seres humanos! La creación no es considerada en su vitalidad, con su propio fin, sino como un medio ulterior para conseguir "lo que se pretende". ¿Lo que pretende quién? No, por cierto quien ha creado los bienes, sino quienes se han apoderado de ellos. Por eso el conjunto de "bienes y recursos" es llamado "el capital" de una persona o empresa. El Capital aparece como el poseedor, convertido así en deidad, deidad que sustituye al creador y que se adora en "el mercado". ${ }^{6}$

Por eso no hay expresión más "anti-creacional" (aunque la utilicen en sus presentaciones contables iglesias de doctrinas creacionistas, o ONG's ecologistas) que la de "recursos humanos", citada explícitamente en los ejemplos del DLE. El ser humano es visto como un "recurso", y no como sujeto creativo. El sujeto es el capital y el ser humano un recurso para incrementar ese capital. La capacidad humana no es don o posibilidad ser reconocida y valorada, sino recurso a utilizar para incremento del capital. Se da una reversión total del sentido de la creación. La cuenta "Capital" dispone de los "recursos humanos" para su crecimiento, pero cuando se dispone para su compensación (salarios) eso va a "gastos".

Como marca Pablo en su consideración de la perversión imperial, "porque lo que de Dios se conoce les es manifiesto, pues Dios se lo manifestó. Porque las cosas invisibles de él, su eterno poder y deidad, se hacen claramente visibles desde la creación del mundo, siendo entendidas por medio de las cosas hechas, de modo que no tienen excusa. Pues habiendo conocido a Dios, no le glorificaron como a Dios, ni le dieron gracias, sino que se envanecieron en sus razonamientos, y su necio corazón fue entenebrecido. Profesando ser sabios, se hicieron necios, y cambiaron la gloria del Dios incorruptible en semejanza de imagen de hombre corruptible, de aves, de cuadrúpedos y de reptiles. Por lo cual también Dios los entregó a la inmundicia, en las concupiscencias de sus corazones, de modo que

\footnotetext{
${ }^{5}$ DLE, ad loc

${ }^{6}$ Ver: Joerg Rieger: El Mercado como religión. Buenos Aires, La Aurora, 2017.
} 
deshonraron entre sí sus propios cuerpos, ya que cambiaron la verdad de Dios por la mentira, honrando y dando culto a las criaturas antes que al Creador, el cual es bendito por los siglos. Amén". (Rom 1:19-25). La deshonra de los cuerpos es, justamente, el desconocimiento del ser humano como creación de Dios, como su imagen, y portador de la dignidad que le da el creador, y su transformación en un recurso humano para llevar a cabo una empresa. Hemos ido más allá de lo que la constatación paulina veía en el Imperio: ahora ni siquiera tiene la imagen de un hombre o una bestia: tiene la imagen " $\$$ ".

El lenguaje refleja la visión del mundo. El lenguaje de la contabilidad capitalista nos muestra su concepción anti-ecológica, en el sentido que no construye la casa sino que la destruye, ya que su fin no es honrar al creador sino enaltecer a la criatura, que en este caso no es siquiera el ser humano (que es un "recurso"), sino el ídolo Mammón, al cual todo es sometido. En ese sentido el capitalismo, no solo en su práctica, sino en su propia concepción del mundo y de la actividad humana, es contrario al sentido de la creación. Por el contrario, la fe bíblica sostiene que la creación es un bien divino, y su objeto es el amor de Dios y no el crecimiento ilimitado del capital.

\section{Cuidado/conservación}

Hay una cierta tensión entre el primer relato de la creación (Gén 1:1-2:3) y el llamado segundo relato (Gén 2:4 ss). Esta tensión está referida, entre otras cosas, al mandato y lugar del ser humano en la creación. Mientras el primer lenguaje es de dominación ( $k b s h)$ y gobierno $\left(r d^{\prime}-1: 28\right)$, en el segundo relato el mandato de Dios al poner al ser humano en el Edén es para trabajar ('bd) y cuidar ( $s h m r-2: 15)$. Siguiendo el principio de interpretar un texto a la luz de otros, debemos suponer que el segundo texto explicita el sentido del mandato del primero. El mandato general de dominar y gobernar se hace concreto en la tarea en el Edén: trabajar (hacer producir, fructificar, incluido en el primer mandato) y cuidar. No le es dada una disposición despótica sobre lo creado. Lo creado tiene vida, y en cuanto le es encomendado al ser humano su gobierno, ese gobierno tiene por finalidad el asegurar esa vida, el facilitarla, el hacer que fluya, es decir, cuidarla.

Sin embargo, en ese cuidar deben considerarse al menos otras dos condiciones: el cuidado de la creación y la vida frente al peligro al que esa vida puede estar expuesta incluye también la vida del ser humano vulnerable. Aquí aparece otra tensión, vinculada con la relación de la "deuda" del ser humano con Dios en virtud de la vida otorgada y consumida. ${ }^{7}$ Dios no solo le da la vida al ser humano, sino que éste, para su sustento cotidiano "consume vida", usa de otras vidas para su propia subsistencia.

${ }^{7}$ Un desarrollo más amplio de este argumento puede verse en: Michel Clévenot: Lectura materialista de la Biblia. Salamanca: Ediciones Sígueme, 1978. 
Ello constituye una "deuda" frente al creador. La teología sacrificial, expresada en el Antiguo Testamento en la tradición sacerdotal, ve el modo de compensar esa deuda en el sacrificio como ofrenda a Dios mediante la quema de una parte de lo consumido (Lev 3 et passim). Por otro lado, la tradición profética ve esa reparación en el cuidado de la vida de los más vulnerables, usando parte de lo que se obtiene por el trabajo sobre la creación para sostener la vida de quienes no pueden disponer de lo necesario por sus propias fuerzas o condición social: la viuda, el huérfano, el pobre, el extranjero. Esta preocupación recorre todo el texto veterotestamentario, incluso en el caso de la Ley, especialmente en Deuteronomio (Deut 14). El resumen lo presentará Oseas: "Misericordia quiero, y no sacrificio" (Os 6:6), texto que usará Jesús en su polémica con los fariseos (Mt 9:13; 12:7). El texto de la escena del juicio mesiánico en Mateo 25:31-46 tiene el mismo sentido. Honrar al creador, cuidar y hacer fructificar la creación y servir al Mesías se concretan en la atención a quienes tienen su vida en riesgo. Una conciencia ecológica que no contemple centralmente la vida de los más débiles no coincide con la comprensión bíblica del cuidado de la creación.

La literatura joanina destaca justamente el hecho del sentido vital de lo creado y de la actividad divina. Junto al Génesis constituyen los escritos bíblicos que más emplean la palabra "vida" en su sentido de don de Dios, fuerza y dinámica, aliento y existencia. El prólogo del Evangelio de Juan (Jn 1:1-18, esp. 1-4) vuelve sobre el hecho creacional destacando el lugar del logos divino como el dador de vida. Esto es necesario para la concepción de la encarnación de la teología joanina. La totalidad de lo vital se hace presente en la encarnación del logos (de allí la expresión de Jesús "Yo soy la vida" -11:25; 14:6). Es la adhesión a ese logos encarnado, en el cual se hace visible el amor creador y vital de Dios, el que da vida (tanto el Evangelio como la Primera Carta de Juan abundan en este tema. Ver, por ejemplo, Jn 6 o 1Jn 5). También en este caso, el cuidar la vida de los más débiles es parte de esta fe y amor del logos en nosotros (1Jn 3:10-17).

La otra condición del ser humano como "guarda de la creación" es que el ser humano interviene activamente en el desarrollo de la vida. No es un simple espectador de cómo ésta se desenvuelve de por sí, sino que también tiene una capacidad de contribuir a esta creación desde su propia iniciativa. La tarea humana no es la de conservar inalterada la creación como la ha recibido, la de ser un guardián de una naturaleza intangible (aunque por momentos deba asumir esa función frente a determinadas circunstancias y lugares). Más allá de la dinámica vital que está inserta en la propia condición de lo creado, le es dado al ser humano potenciar esa dinámica, alterarla, desarrollarla aun fuera de sus carriles "naturales". No hay pecado en ello. El pecado, (en el sentido original de la palabra, desviarse del objetivo o propósito) está en desviarse de la labor productiva, re-creadora, con-creadora, para destruir la creación a favor de otras deidades idolátricas: Molok, Mammón, o El Capital. 
Por otro lado, cierta concepción conservacionista que ve a la naturaleza como una deidad en sí misma, cae en un cierto tipo de idolatría tanto como quienes se dan a la destructiva tarea de alterar lo creado, no para sustento de la vida sino para enriquecimiento de los poderosos. Una cosa es el respeto y cuidado de la tierra en su dinámica vital y todo lo creado como parte del mandato divino, y otra cosa es su endiosamiento, una deificación de "lo natural". Hay un esfuerzo en el relato bíblico de confrontar justamente la idea de la divinización de las potencias naturales. Aun estas se deben al Creador, y Pablo debe combatir la idea de un sometimiento a "los elementos" 8 , tanto como el atarse a la ley, como una amenaza a la libertad cristiana (Gál 4:3, 9; Col 2:20).

Esto puede resultar polémico frente a algunas líneas teológicas principalmente en las teologías de los pueblos originarios o el llamado "ecofeminismo" - que ponderan positivamente cierta valoración de "la tierra" y su culto como deidades, sea como la Pachamama, en un caso, o Gaia en el otro. También puede sonar discordante frente al llamado a "re-encantar" la tierra. No es mi propósito desacreditar estos reclamos, necesarios frente al abuso de una economía utilitarista que desconoce el sentido y vitalidad de la tierra. Sin duda, en el propio relato bíblico la tierra y las aguas son productores de vida (Gén 1:11-12; 20), y en ese sentido participan de la actividad divina de la creación. Lo que quiero señalar aquí es que no se constituyen "de por sí" en una deidad o en potencias separadas del propósito amoroso de la creación, sino que son parte del mismo.

En ese sentido, la fe bíblica nunca es "conservadora": nos presenta una divinidad siempre abierta a lo nuevo, incluso creadora de lo nuevo. Por eso la nueva creación es parte de la promesa divina y de la actividad mesiánica (2Co 5:17). Sin embargo esa nueva creación, y esa nueva humanidad como parte de ella, no prescinden de la primera creación ni de la historia humana. No es lo que Dios ofrece a cambio de la primera creación, sino su re-creación en vista de la actividad destructiva de quienes han optado por otras deidades de muerte. El Dios bíblico no es un Dios conservacionista, sino un Dios renovador, y que invita al ser humano, por la fe, a entrar en esa labor re-creadora.

\section{Dominar/laborar}

Justamente por ello, el trabajo forma parte de la actividad humana. Y sin embargo está marcado por la condición ambigua de la propia humanidad, como creador y consumidor a la vez. El trabajo productivo, como ya hemos visto, está en el mandato originario dado en el Edén, y no solo como "castigo por el pecado", sino como parte de la condición que nos

\footnotetext{
${ }^{8}$ Probablemente esto pueda referirse tanto a los elementos de la tradición presocrática de la cultura helénica (aire, agua, tierra y fuego) como a las potencias astrales de los cultos mesopotámicos, sincretizados, en algunos casos, en los cultos mistéricos vigentes en el período romano.
} 
hace humanos. El "trabajo como castigo" es el trabajo alienado, el que se hace forzado por las condiciones de subsistencia o por las imposiciones de la explotación. Pero el trabajo creativo es lo que nos expresa como humanidad. En un llamativo pasaje el propio Espíritu de Dios aparece como inspirador del trabajo artesanal y artístico (Ex 31:1-11).

El dominio, entonces, no debe ser visto como la autorización para disponer arbitrariamente, sino la facultad de elaborar creativamente. El mandato dado al ser humano de dominar y gobernar en la creación es la condición de posibilidad de su trabajo. Sin esa capacidad de indagar en lo creado, de conocer las características y modalidades de los diversos elementos y potencias activas en la creación, y de hacer un uso de las mismas, la tarea humana, como artífice y productor sería imposible. Pero siempre a partir de la conciencia de lo que pone en juego son "bienes", y no "recursos". Que lo que utiliza en su esfuerzo productivo no será luego desechable, sino parte de la finalidad de su propia producción, y que ello implica una "co-laboración", tanto con otros seres humanos como con otras potencialidades. En eso el ser humano deja de ser un ser "natural" y pasa a ser cultural. Y en ello tiñe de ambigüedad su propia labor, porque la cultura es siempre portadora de dominios y gobiernos en otro sentido, en el de disponer e imponer. Es parte de la paradoja de nuestra existencia que lo que nos posibilita la vida es a la vez lo que abre el camino de la muerte.

En esa paradoja entra el inescapable tema del consumo. El ser humano no puede vivir sin consumir, pero lo que consume es siempre vida. El ser humano crea lo que necesita, para luego necesitar lo que ha creado. Y así, en toda tarea constructiva, donde elabora lo que ha de sostenerlo con vida, afecta otras vidas, tanto humanas como del conjunto de la creación. $Y$ aquí entramos en otro tema complejo, que excede el limitado sentido lexical de este artículo, que es la tensión entre necesidad y deseo. ${ }^{9}$ ¿Hasta qué punto lo que producimos y consumimos responde a nuestra necesidad y al mandato co-creador de Dios, y hasta qué punto son guiados por el "deseo cautivo", lo que Pablo llama "el deseo de la carne", que hace al cautiverio y corrupción de la creación?

El reproche de algunas concepciones ecologistas ha entendido que cierta lectura del mandato bíblico de dominar y gobernar la creación está a la base de los excesos destructivos que hoy ponen en peligro la vida en el planeta. Sin duda algo de cierto hay en ello. Pero una lectura más atenta de los textos nos mostrará que la destrucción no proviene del mandato divino sino de su desobediencia, del sentido de una dominación sin amor al creador ni respeto por lo creado, de una instrumentalización idolátrica que desconoce el sentido de lo bueno y de la labor humana como partícipe del bien.

\footnotetext{
${ }^{9}$ Ver, en este sentido, entro otros, Jung Mo Sung: Deseo, mercado y religión, Santander, Sal Tarrae, 1999.
} 


\section{Para seguir pensando}

En este breve e inacabado repaso del vocabulario bíblico sobre la creación he procurado destacar lo que, a mi entender, posiciona a la fe bíblica en el conjunto de los esfuerzos cooperativos por poner en la conciencia de la actualidad algunas de las problemáticas que hacen realidad ecológica del planeta. Siendo una mirada sobre el texto bíblico no hemos hecho referencia a otras expresiones significativas provenientes del cristianismo hoy, como pueden ser el documento del Consejo Mundial de Iglesias "Juntos por la vida" o la encíclica papal de Francisco "Laudato si", entre otras muchas expresiones en ese sentido.

Lo que hemos querido señalar es que, más allá de las asincronías culturales y distancia hermenéutica, el lenguaje bíblico impone, de por sí, una mirada crítica sobre la teoría y gestión económicas del imperio del capitalismo financiero y sus prácticas consumistas. Tampoco concuerda, sin embargo, con una mirada puramente conservacionista. La preocupación por la integridad de la creación es a la vez una mirada sobre las condiciones de la realidad humana y las injusticias de un orden destructivo, de una acumulación sin precedentes, de una explotación inmisericorde de la potencia de lo creado. Las ciencias del ambiente aportan elementos que nos ayudan a comprender las dinámicas de esa creación y nos alertan de las consecuencias de nuestras acciones, pero quedan cortas en su alcance si no ven en ello la posibilidad de la renovación liberadora del dispositivo mesiánico, inserto en la propia encarnación del logos, "porque también la creación misma será libertada de la esclavitud de corrupción, a la libertad gloriosa de las hijas e hijos de Dios" (Rom 8:21). 